\title{
A pocket of very high suicide rates in a non-violent, egalitarian and cooperative population of South-East Asia
}

\author{
F. Jollant ${ }^{1 *}$, A. Malafosse ${ }^{2}$, R. Docto $^{3}$ and C. Macdonald ${ }^{4}$ \\ ${ }^{1}$ McGill University, Department of Psychiatry; and Douglas Mental Health University Institute, McGill Group for Suicide Studies, Montréal \\ (Québec), Canada \\ ${ }^{2}$ Department of Psychiatry, Genetics Laboratory, Academic Hospital of Geneva, Hôpital Belle-Idée, Chêne-Bourg, Switzerland \\ ${ }^{3}$ Palawan State University, Puerto Princesa City, Philippines \\ ${ }^{4}$ Unité d'Anthropologie Bioculturelle, National Center for Scientific Research (CNRS), Marseille, France
}

Background. Extremely high rates of suicide localized within subgroups of populations where suicide is rare have been reported. We investigated this intriguing observation in a population of South-East Asia, where local culture should theoretically be preventative of suicide.

Method. A team including an anthropologist and a psychiatrist surveyed all cases of suicide that had occurred over 10 years in four isolated regions. A psychological autopsy was carried out comparing each suicide case with two matched control cases.

Results. In a region of 1192 inhabitants, 16 suicides occurred, leading to an annual suicide rate of 134/1000 00 which is 10 times the rate in the USA or Canada. By contrast, three ethnically similar distant communities showed low to null rates. The gender ratio was three males to one female and two-thirds of cases were aged below 35 years. Methods of suicide were poisoning and hanging and motives mainly included interpersonal discord. The pattern of developmental and clinical risk factors was somewhat different from Western countries, showing no childhood maltreatment, only one case of alcohol/substance abuse and impulsive-aggressive personality but elevated rates of social anxiety. Suicide cases had very high frequencies of second-degree biological relatives who committed suicide.

Conclusions. Our study confirms a persistent phenomenon of high suicide rates restricted to a subgroup of a preindustrialized population. We hypothesized this might be explained by isolation and endogamy, which may have promoted the selection/amplification of genetic vulnerability factors, or a contagion effect. These findings shed light on suicide from both a singular and a universal perspective, suggesting that particular local conditions may significantly modulate the rate of this complex behavior.

Received 30 August 2013; Revised 5 December 2013; Accepted 7 December 2013; First published online 10 January 2014

Key words: Culture, genetics, human behavior, indigenous people, suicide.

\section{Introduction}

Suicide is a universal human phenomenon. However, it occurs at various frequencies according to location. Suicide rates range from below 10/100000 inhabitants in Southern Europe and in most developing countries, including the Philippines, up to $30-40 / 100000$ in Hungary or Lithuania (Hawton \& van Heeringen, 2009). Although cultural, genetic and methodological

\footnotetext{
* Address for correspondence: Dr F. Jollant, Douglas Mental Health University Institute, 6875 boulevard LaSalle, Montréal, Québec H4H 1R3, Canada.

(Email: fabrice.jollant@mcgill.ca)

These findings of this paper were presented at the International Academy for Suicide Research (IASR) meeting in Montreal, Canada, on 12 June 2013.
}

hypotheses have emerged, the origins of these variations are largely unknown.

To address some of these issues, we report here an investigation of suicide among a non-violent, cooperative and egalitarian indigenous population living on the island of Palawan in the Philippines. The Palawan people form an ethnolinguistic entity of approximately 40000 to 50000 inhabitants (Macdonald, 2007). Forty years of anthropological study of this population by C.M. has led to the unexpected observation of a very high frequency of suicide cases, between 130 and 170, among a small subgroup of Palawans (to protect this population, we have limited references to locations) (Macdonald, 2007). This phenomenon seems to have existed within this subgroup for several decades. By contrast, neighboring Palawan subgroups of similar culture report suicide very rarely. The first aim of this 
study was therefore to assess the persistence of this intriguing phenomenon over the past decade, and compare suicide rates in the aforementioned region to rates in three distant control Palawan regions.

The second objective of our study was to investigate risk factors for suicide in this population. Suicide risk factors have mainly been studied in modern Western societies, limiting the generalizability of findings. Risk factors usually include both proximal (e.g. negative life events, mental disorders) and distal factors (e.g. childhood abuse, genetic factors) (Hawton \& van Heeringen, 2009). A generally accepted model is that proximal factors act as triggers on pre-existing vulnerabilities (Mann, 2003).

A previous anthropological investigation of suicide among the high-risk subgroup of Palawans indicated the absence of many classical risk factors, including childhood maltreatment and alcohol/substance abuse. However, no systematic structured assessment of these risk factors (including psychiatric disorders) has been performed. Therefore, we conducted standardized psychological autopsies of all suicide cases reported within this subgroup over the past 10 years.

Apart from the logistic complexity and physical challenge of such studies, studying relatively isolated populations presents interesting advantages (Shifman \& Darvasi, 2001), notably (1) a genetically and culturally homogeneous population living in a uniform environment, (2) little migration and thus more intact families, allowing good genealogical records, and (3) the possibility of investigating a pre-industrialized non-Western population having limited contact with modern lifestyles.

On the basis of previous investigations, we hypothesized that (1) suicide would remain at a very high rate in the aforementioned region in comparison to the three control regions and (2) risk factors for suicide in this population would differ, in part, from those reported in Western countries.

\section{Method}

A unique international team including a psychiatrist, an anthropologist, a geneticist and local scholars organized this project. The field missions were conducted in 2012 and 2013.

\section{Ethics}

This study was approved by the Provincial Health Office, Puerto Princesa City and the National Commission on Indigenous Peoples, Quezon City, Philippines. In addition, the Douglas Institute Research Ethics Board conducted a scientific assessment of the project. An information sheet and consent forms were translated into both Tagalog (the official language of the Philippines) and Palawan. In all three villages investigated, the protocol was first explained to, and then approved by, the tribal councils, who gave their signed consent (signature or thumb mark). Then, each main participant gave their signed informed consent prior to assessment.

\section{Census of the total population and suicide cases}

Our investigation focused on three villages of the region previously shown to have a very high rate of suicide (Macdonald, 2007). These villages have a close relationship with each other, with an almost complete absence of non-Palawan immigrants. Travel to these villages takes 1 day from Puerto Princesa, the main city of Palawan island, and includes car, motorcycle, walking and buffalo. The climate is tropical and the region is in a malaria-resistant zone.

We conducted our own census of the investigated villages to determine the number of inhabitants per household and their gender. Because we had limited time, we did not determine age in the census. We counted 328 inhabitants (175 males, 153 females) in village A, 499 (256/243) in B and 365 (192/173) in C, making a total population of 1192 inhabitants. A previous raw census in 2002 suggests a small increase in population over the past 10 years. However, considering the lack of precise statistics, we opted to keep our current population number as the base rate for the calculation of the 10 -year suicide rate, with the risk of underestimating this rate.

As no official registry exists, data on all suicide cases that occurred from June 2002 to May 2012 in the three villages were collected from multiple local informants. Data on suicide cases before June 2002 were collected and published previously (Macdonald, 2007) and were therefore excluded with confidence.

\section{Control regions}

We also conducted an investigation of three distant Palawan regions as controls. Reports of suicide cases during the same period were collected by the same methods described earlier. However, for the control regions, census of the related population was based on administrative sources and not on direct counting because of time restrictions.

\section{Psychological autopsy}

All suicide cases from the target region were assessed by one or more informants who were well acquainted with the victim. Importantly, as the Palawan people have very close relationships with one another, highly concordant information from different sources was 
almost always provided, enabling cross-validation of the information obtained. No medical records or notes left by the victims were available to complete the assessment because of lack of medical services and illiteracy.

Each suicide case was matched with two controls of the same sex and in the same age group. All controls came from the same three villages. Great care was taken to exclude controls who were close relatives (i.e. less than three genetic degrees, which also represents the incest threshold) of the investigated suicide cases or other controls to avoid overestimation of family genetic risk. Each control was assessed by one or more informants using the same proxy-based procedure as for the assessment of suicide completers. This method has been used with success in numerous previous psychological autopsies (Dumais et al. 2005). We had no direct contact with the controls during the assessment. All of the informants contacted agreed to participate.

For each case, a genealogical tree was constructed to detect cases of suicide, suicide attempt, 'madness' or 'mental deficiency' in the family, and to assess the degree of closeness with other cases assessed.

As there were no Palawan psychiatrists or psychologists, the main psychiatric diagnoses were carried out by F.J., a psychiatrist, with the help of C.M., an anthropologist who speaks Palawan fluently, has written the only existing Palawan-Tagalog-English dictionary (www-01.sil.org/asia/philippines/works-pal. html) and has a deep knowledge of local concepts including the expression of emotions and feelings. A Palawan translation of the Mini-International Neuropsychiatric Interview (MINI) 6.0.0 (Sheehan et al. 1998) was provided by C.M., with the help of two native Palawan people. A back-translation was then provided by C.M. and verified by F.J. to ensure adequacy with the original version. All diagnoses were current for the controls and at the time of death of the suicide victims.

Impulsive-aggressive personality disorders were clinically assessed by systematically asking about a personal history of self or externally directed aggression, theft, incarceration, interpersonal arguments or anger propensity. A checklist was used for all other variables, as shown in Table 1. Sociodemographic variables were obtained by interviewing informants. A suicide attempt was defined as 'any act carried out with some intent to die', as defined by Mann (2003). Early parental loss was defined as the loss of one parent before the age of 15 years. The population usually discriminates between 'madness' and 'mental deficiency', the latter term referring to intellectual disability. Although they lack precise definition, we chose to keep these terms given by informants because, in many cases, the individual to whom they were referred was not sufficiently known by the informant to clarify the diagnosis.

\section{Statistical analyses}

The suicide rate was calculated as the number of suicides over 10 years, divided by 10 for an average annual number, divided by the total population (1192) and multiplied by 100000 .

Statistical analyses were carried out using SPSS version 20 (SPSS Inc., USA). Univariate analyses were conducted first. Comparisons of quantitative data between the two groups were made by means of a Mann-Whitney test because of the small size of the groups. Associations between qualitative variables and groups were calculated with the $\chi^{2}$ test. Variables significantly associated with group at the univariate stage were then separately entered into a logistic regression model for multivariate analysis, using a stepwise procedure. The $p$ level was set a priori at 0.05 .

\section{Results}

\section{Suicide rates and description of cases}

Sixteen suicides were reported between June 2002 and May 2012, giving a global suicide rate of 134/100000 inhabitants per year. Suicide victims were 12 males and four females (giving a gender ratio of 3/1) and suicide rates were 193/100000 in males and 70/100000 in females. All suicides occurred between 2004 and 2011.

We could not find any close informant for one suicide case (male, $\sim 40$ years old) who seemed to have lived surprisingly isolated. Therefore, the psychological autopsy was conducted on 15 suicide completers and 30 controls.

The main method of suicide was poisoning by tuba (12 cases), a vegetal poison produced from a readily available plant (genus Derris) traditionally used to hunt and fish. The three remaining cases hanged themselves. One man first killed his pregnant wife before committing suicide.

Reported motives were marital conflicts/separation (six cases), painful disease (severe migraines; painful and intractable tongue lesion, possibly cancer; and painful motor handicap; three cases), poverty or debt (three), death of husband (one), sentimental rebuttal (one) and unknown (one).

Nine suicide victims were aged between 15 and 24 years $(56.25 \%)$, two between 25 and 34 years $(12.5 \%)$, three between 35 and $44(18.75 \%)$ and two between 55 and 64 (12.5\%).

Among the suicide completers assessed, four were close relatives (three siblings and one first cousin) and 
Table 1. Comparison of sociodemographic and clinical variables between suicide completers and controls matched for gender and age

\begin{tabular}{|c|c|c|c|c|}
\hline & $\begin{array}{l}\text { Suicide } \\
(n=15)\end{array}$ & $\begin{array}{l}\text { Controls } \\
(n=30)\end{array}$ & $\begin{array}{l}\text { Mann-Whitney } \\
U \text { or } \chi^{2}\end{array}$ & $p$ \\
\hline Early parental loss & $7(46.7)$ & $7(23.3)$ & 2.5 & 0.1 \\
\hline Childhood maltreatment & $0(0)$ & $0(0)$ & - & - \\
\hline Attended elementary school & $2(13.3)$ & $8(26.7)$ & 1.0 & 0.3 \\
\hline Marital status (married) & $10(66.7)$ & $15(50.0)$ & 1.2 & 0.5 \\
\hline Number of children & $1(0-6)$ & $1(0-10)$ & 204.0 & 0.6 \\
\hline Financial debts & $4(26.7)$ & $9(30.0)$ & 0.05 & 0.8 \\
\hline Current physical disease & $7(46.7)$ & $7(23.3)$ & 2.5 & 0.1 \\
\hline Depressive disorders & $4(26.7)$ & $2(6.7)$ & 3.5 & 0.06 \\
\hline Bipolar disorders & $0(0)$ & $2(6.7)$ & 1.0 & 0.3 \\
\hline Panic disorder & $2(13.3)$ & $0(0)$ & 4.2 & 0.04 \\
\hline Agoraphobia & $5(33.3)$ & $3(10.0)$ & 3.7 & 0.05 \\
\hline Social phobia & $7(46.7)$ & $4(13.3)$ & 6.0 & 0.01 \\
\hline PTSD & $0(0)$ & $0(0)$ & - & - \\
\hline Generalized anxiety & $0(0)$ & $0(0)$ & - & - \\
\hline Alcohol abuse & $1(6.7)$ & $0(0)$ & 2.0 & 0.2 \\
\hline Substance abuse & $0(0)$ & $0(0)$ & - & - \\
\hline Tobacco use & $7(46.7)$ & $13(43.3)$ & 0.04 & 0.8 \\
\hline Psychotic disorder & $0(0)$ & $0(0)$ & - & - \\
\hline Impulsive-aggressive PD & $1(6.7)$ & $0(0)$ & 2.0 & 0.2 \\
\hline Expressed suicidal ideas (lifetime) & $0(0)$ & $5(16.7)$ & 2.8 & 0.09 \\
\hline Previous suicide attempt & $1(6.7)$ & $1(3.3)$ & 0.3 & 0.6 \\
\hline \multicolumn{5}{|l|}{ Family history of suicidal act } \\
\hline First genetic degree & $9(60.0)$ & $4(13.3)$ & 10.6 & 0.001 \\
\hline At least second degree & $12(80.0)$ & $9(30.0)$ & 10.0 & 0.002 \\
\hline At least third degree & $13(86.7)$ & $20(66.7)$ & 2.0 & 0.2 \\
\hline $\begin{array}{l}\text { Family history of 'madness' or } \\
\text { 'intellectual deficiency' }\end{array}$ & $8^{\mathrm{a}}(61.5)$ & $17(56.7)$ & 0.09 & 0.8 \\
\hline
\end{tabular}

PTSD, Post-traumatic stress disorder; PD, personality disorder.

${ }^{a}$ No clear data for two cases.

Values given as number (percentage) or median (range).

Diagnoses are at time of death for suicide victims and current for controls.

two others were first cousins. Other cases were not directly related to each other.

In the first control region, two suicides occurred over the past 10 years among a population of 1500 inhabitants, leading to a suicide rate of 13.3/100000 inhabitants. In the most extreme underestimation of the number of suicide cases by $100 \%$ and overestimation of the population size by $100 \%$, this would lead to a suicide rate of 54/100000 well below the rate in the target subgroup. In the two other control communities, no suicide could be detected among a population of 1000-1500 inhabitants each over the past 10 years.

\section{Psychological autopsy}

Higher rates of panic disorder, agoraphobia and social phobia, and first- and second-degree biological relatives who committed suicide were found in suicide completers compared to controls (Table 1). There was also a trend toward increased depressive disorders within this group. Social phobia, but not panic disorder and agoraphobia, remained significant when depression was taken into account in the logistic regression (social phobia: Wald $=3.5, p=0.06$; depression: Wald $=1.0, p=0.3$ ).

The two groups did not differ in terms of other variables. Importantly, no case of childhood maltreatment and only one case of alcohol substance abuse and impulsive-aggressive personality disorder was detected in either group.

A burden of risk in terms of number of psychiatric disorders was calculated for each individual as the sum of all available diagnoses (among 11 possible). Between-group comparison showed a significantly higher number of diagnoses in suicide victims versus controls [median $=2$ (range $0-4$ ) vs. 1 (range $0-4), U=109.5, p=0.004]$. 


\section{Discussion}

Suicide is usually considered to be extremely low in pre-industrialized populations, leading to the notion that this is a problem of modern societies. Although this may be true in many instances, we report here a suicide rate of $134 / 100000$ inhabitants, that is 10 times the rate of suicide in Canada, the USA and many European countries, among a small subgroup of indigenous people in the Philippines. This extremely high suicide rate seems to have persisted for the past decade (and probably over the past 50 years) and, importantly, is very localized. Indeed, the rest of the Palawan population, of similar culture, does not seem to share this feature.

Hence, in this subgroup suicide does not seem to be related mainly to cultural factors. Indeed, the social organization of this population should theoretically represent a protective factor against suicide. For instance, Palawans show a high degree of family ties, frequent interactions and a sense of community. Cooperation is a core principle of societal functioning. Violence is avoided and Palawans are usually described as a shy people, with no rigid code of honor, who value escape when facing aggression. Furthermore, the group will identify a leader based on that person's ability to settle disputes. In this egalitarian population, the status of leader entails no power or privilege. Moreover, Palawan culture does not promote suicide as a way to solve problems and Palawans usually express perplexity and fatality regarding suicide. Finally, this population has been living more or less in similar conditions for many years and, so far, has not suffered brutal displacement, forced assimilation or 'Westernization'. Other reasons have, therefore, to be sought.

Of note, other similarly striking differences in rates of suicide among identical ethnolinguistic groups have previously been reported worldwide in the ethnological literature (Macdonald, 2007), including the Baruya (suicide rate $=96 / 100000$ ) versus the Ankave (almost none) in New Guinea (Bonnemère, 1992) and the Aguarunas (suicide rate $=180 / 100000$ ), a subgroup of the Jivaro people (very low suicide rates) (Brown, 1986). These populations differ between them in several respects from the use of violence to gender relationships (symmetry versus asymmetry) or the structure of society (egalitarian versus hierarchical) (Macdonald, 2007). However, all of these populations share the common feature of a high degree of isolation and endogamy (Macdonald, 2007), a factor that may play a significant role in this high rate of suicide. This could explain the significantly higher frequency of suicide cases among first and second biological relatives of suicide completers (reaching
$80 \%$ of cases). Of note, the family rate of suicide is also very high among controls, up to almost $70 \%$ at the third biological degree. Unfortunately, we do not have a precise index of endogamy. Therefore, at this stage, its role remains theoretical. Future investigation of this population will attempt to measure this factor.

Two non-mutually exclusive hypotheses may be drawn from this. First, this high level of isolation may increase the risk of contagion effect, a recognized phenomenon in suicidal behavior (Brent \& Melhem, 2008). However, this phenomenon is usually limited in time. A second explanation may be that an original founding effect or a genetic drift (Stone et al. 2006) has selected particular alleles conferring a higher risk of suicide, the frequency of which is amplified by isolation. Genetic factors have been robustly implicated in the vulnerability to suicide in family, twin and adoption studies (Brent \& Mann, 2005). These genetic factors may render carriers more sensitive to stressful events and more likely to experience pain, hopelessness and suicidal ideas, and to act upon these negative thoughts. More investigations are necessary to identify these factors.

Many suicide risk factors described in Western populations are absent here, suggesting a particular developmental and clinical profile, in comparison to the 'suicidal trajectories' previously described in the literature (Seguin et al. 2011). One important risk factor is childhood maltreatment, previously found in up to $30 \%$ of suicide cases in Western countries (Turecki et al. 2012) but in none of the suicide and control cases in Palawan. External informants and prolonged observation tend to confirm the absence of childhood maltreatment in this population. Moreover, Palawan people are known to be non-violent and children are usually raised by an extended family. This may reduce the risk of childhood abuse and consequently the risk of transmission of these behaviors across generations.

Mental disorders are major risk factors for suicide (Cavanagh et al. 2003), which is also the case in the Palawan subgroup, with a higher burden of risk in suicide victims. However, the profile of mental disorders found here differs from those previously described in Western studies, with the exception of increased depression. This is notably the case for social phobia, which was associated with suicide independently of depression. Although this association has been described previously (Kanwar et al. 2013), it seems to be a particular signature of Palawan suicide, accounting for half of all suicide cases. It is important to mention that Palawan people tend to be shy in general. Nevertheless, people clearly identified individuals with social phobia as different from being simply shy (e.g. 'he never ate with the group during feasts' or 'she would never gather with us to discuss in group'). 
It is noteworthy that motives for suicide are not unique to this population (Foster, 2011), with interpersonal conflicts accounting for almost half of cases, followed by painful diseases. Poverty and debt may also be in play through a feeling of failure to take care of family. The 'social feature' of suicide is therefore also found among the Palawans, and social phobia may play a significant role in suicidal tendencies by making some individuals more sensitive to their social environment and less likely to seek help from others.

Alcohol and drug abuse were not significant risk factors, mainly because these substances are not readily available. In addition, we could not identify any impulsive or aggressive personality disorders among the studied groups, with the exception of one individual who had alcohol problems, a long history of arguments with neighbors and who killed his wife before committing suicide. The lack of these major risk factors again supports the idea of a different developmental trajectory to suicide. Psychotic and bipolar disorders were rarely reported in our sample, possibly because of the small sample sizes.

Suicide attempts, although reported, are rare in this population. As a consequence and contrary to what is found in Western countries, a history of suicide attempt is not a risk factor of future suicide in this population, which is significant in the perspective of prevention. Many explanations may be put forward, including the high lethality of the means used being associated with the lack of closed medical settings, the absence of available medications, which is the primary method used in non-fatal acts, and the lack of available medical services, which has been incriminated as facilitating acts carried out as a 'cry for help'.

To our knowledge, only two other studies have examined risk factors for suicide in endogamous populations. The first one (Egeland \& Sussex, 1985) studied the Old Order Amish in Pennsylvania. Like the Palawans, the Amish are non-violent, show high social cohesion, live in isolation, and show little use of alcohol and drugs. Contrary to the Palawans, however, suicide among the Amish is taboo and rates of suicide have remained low over the past 100 years. Suicide was associated with mood disorders, although it was found to cluster in some families, while other families showed high rates of mood disorders without suicide. We could not assess this latter aspect from our data, and this constitutes a suitable topic for future research. The second study involved an endogamous Arab kindred in Israel that showed high rates of suicide (around 67/100000) but also suicide attempts (Hamdan et al. 2011). Like the Palawan suicide completers, suicide attempters in this population showed high levels of depression and anxiety, high frequencies of first-degree relatives who attempted suicide, and low rates of alcohol and substance abuse. However, they also showed high levels of impulsive aggression and childhood abuse. In these two groups and in the Palawans, endogamy, family transmission and higher burden of mental disorders seem to be major risk factors, although the precise picture varies from one group to another.

Our study has several limitations. First, language barriers may limit the reliability of some psychiatric diagnoses. However, we believe that many concepts, such as low mood or social phobia, were perfectly translated and understood in Palawan. We also took great care to ensure that cultural factors were taken into consideration when determining disorder threshold. Second, as in many studies, recall issues may bias some data collection. This issue is probably exacerbated by the fact that Palawans do not make written records of any event. Nonetheless, the habit of relying on their memory and the fact that many informants were usually present during interviews may have limited this effect. Third, suicide was not confirmed by any medical authorities, nor was there any evidence of suicide notes. Therefore, false positives may have altered the accuracy of certain findings. Fourth, although the control villages were similar in size and culture to the high-risk villages, we cannot exclude the possibility that they differ from each other by factors we did not measure. However, our long-time observations suggest that the rate of suicide is usually low among the Palawans. Finally, as many informants were usually present during interviews, it cannot be excluded that a desirability effect may have biased some reports. Future psychological autopsies in this population may be conducted in different conditions to examine the importance of this effect in this otherwise very social population.

We hope that the unique investigation reported here will shed light on the existence of isolated groups at very high risk of suicide across the world, and increase our understanding of suicide from both a singular (e.g. low level of impulsivity, violence, childhood abuse and alcohol/drug use; high level of anxiety; the potential effect of isolation among Palawans) and a universal (e.g. family history of suicide and personal burden of mental disorders) perspective.

\section{Acknowledgments}

F.J. received a Fond de Recherche du Québec Santé (FRQS) salary grant and C.M. a CNRS travel grant. The study sponsors had no role in designing the study, collecting data, writing the report or in the decision to submit the report.

We express our sincere gratitude to Edie and Botoy for their help in translating the consent forms, 
questionnaires and instructions into the Palawan language, to Edie and his wife for hosting us during several weeks, and to the Palawan people for welcoming us and for their genuine interest in participating in the study. F.J. thanks his colleagues for taking care of his patients during his long leave in the field. We thank C. Nagy for her valuable help in editing the manuscript.

\section{Declaration of Interest}

None.

\section{References}

Bonnemère P (1992). Suicide et homicide: deux modalités vindicatoires en Nouvelle-Guinée. Stanford French Review 16, 19-43.

Brent DA, Mann JJ (2005). Family genetic studies, suicide, and suicidal behavior. American Journal of Medical Genetics. Part C, Seminars in Medical Genetics 133, 13-24.

Brent DA, Melhem N (2008). Familial transmission of suicidal behavior. Psychiatric Clinics of North America 31, 157-177.

Brown MF (1986). Power, gender, and the social meaning of Aguaruna suicide. Man 21, 311-328.

Cavanagh JT, Carson AJ, Sharpe M, Lawrie SM (2003). Psychological autopsy studies of suicide: a systematic review. Psychological Medicine 33, 395-405.

Dumais A, Lesage AD, Alda M, Rouleau G, Dumont M, Chawky N, Roy M, Mann JJ, Benkelfat C, Turecki G (2005). Risk factors for suicide completion in major depression: a case-control study of impulsive and aggressive behaviors in men. American Journal of Psychiatry 162, 2116-2124.
Egeland JA, Sussex JN (1985). Suicide and family loading for affective disorders. Journal of the American Medical Association 254, 915-918.

Foster T (2011). Adverse life events proximal to adult suicide: a synthesis of findings from psychological autopsy studies. Archives of Suicide Research 15, 1-15.

Hamdan S, Melhem N, Orbach I, Farbstein I, El-Haib M, Apter A, Brent D (2011). Risk factors for suicide attempt in an Arab kindred. Journal of Affective Disorders 132, 247-253.

Hawton K, van Heeringen K (2009). Suicide. Lancet 373, 1372-1381.

Kanwar A, Malik S, Prokop LJ, Sim LA, Feldstein D, Wang Z, Murad MH (2013). The association between anxiety disorders and suicidal behaviors: a systematic review and meta-analysis. Depression and Anxiety 30, 917-929.

Macdonald CJ-H (2007). Uncultural Behavior: An Anthropological Investigation of Suicide in the Southern Philippines. University of Hawai'i Press: Honolulu.

Mann JJ (2003). Neurobiology of suicidal behaviour. Nature Reviews Neuroscience 4, 819-828.

Seguin M, Renaud J, Lesage A, Robert M, Turecki G (2011). Youth and young adult suicide: a study of life trajectory. Journal of Psychiatric Research 45, 863-870.

Sheehan DV, Lecrubier Y, Sheehan KH, Amorim P, Janavs J, Weiller E, Hergueta T, Baker R, Dunbar GC (1998). The Mini-International Neuropsychiatric Interview (M.I.N.I.): the development and validation of a structured diagnostic psychiatric interview for DSM-IV and ICD-10. Journal of Clinical Psychiatry 59 (Suppl. 20), 22-33; quiz 34.

Shifman S, Darvasi A (2001). The value of isolated populations. Nature Genetics 28, 309-310.

Stone L, Lurquin PF, Cavalli-Sforza LL (2006). Genes, Culture, and Human Evolution: A Synthesis. Wiley-Blackwell: Chichester.

Turecki G, Ernst C, Jollant F, Labonte B, Mechawar N (2012). The neurodevelopmental origins of suicidal behavior. Trends in Neurosciences 35, 14-23. 